\title{
Prevalence and Associated Factors of Diabetic Retinopathy among Type 2 Diabetes Mellitus Patients in Brunei Darussalam: A Cross-sectional Study
}

\author{
Sajid Adhi Raja', Vui Heng Chong ${ }^{2}$, Noor A. Rahman ${ }^{1,3}$ Lilabi M P Shakir $^{4}$, Joe Knights ${ }^{5}$ \\ ${ }^{I}$ Department of Ophthalmology, Pengiran Muda Mahkota Pengiran Muda Haji Al-Muhtadee Billah, Tutong Hospital, Tutong, Brunei \\ Darussalam \\ ${ }^{2}$ Department of Medicine, RIPAS and PAPRSB IHS Pengiran Muda Mahkota Pengiran Muda Haji Al-Muhtadee Billah, Tutong Hospital, \\ Tutong, Brunei Darussalam \\ ${ }^{3}$ Department of Ophthalmology, RIPAS Hospital, Bandar Seri Begawan, Brunei Darussalam \\ ${ }^{4}$ Department of Community Medicine, Calicut Medical College, Kerala, India \\ ${ }^{5}$ Pengiran Anak Puteri Rashidah Sa'adatul Bolkiah Institute of Health Sciences, Universiti Brunei Darussalam, Gadong, Brunei Darussalam
}

Purpose: To determine the prevalence of diabetic retinopathy (DR) and the factors associated with retinopathy among type 2 diabetes mellitus (DM) patients in Brunei Darussalam.

Methods: Cross-sectional study of all type 2 DM patients who attended diabetic eye screening over a 3-month period at one of four government hospitals. We assessed association between DR with the following variables: age, sex, glycated hemoglobin ( $\mathrm{HbA1c})$, duration of $\mathrm{DM}$, hypertension, hyperlipidemia, and microalbuminuria.

Results: There were 341 patients (female, 58.9\%; mean age, $55.3 \pm 11.9$ years) with a mean duration of DM of $9.4 \pm 7.4$ years and mean serum $\mathrm{HbA} 1 \mathrm{c}$ of $8.4 \% \pm 1.9 \%$. The overall prevalence of any DR was $22.6 \%$ (95\% confidence interval, 18.8-27.1) with prevalence rates of $4.1 \%$ (95\% confidence interval, 2.1-6.4) for proliferative DR and 9.7\% (95\% confidence interval, 6.8-13.2) for vision-threatening DR. Multivariate analysis showed that DR was significantly associated with certain age groups (reduced in older age groups), longer duration of DM (11 years or more), poor control (HbA1c $>9.0 \%$ ) and presence of any microalbuminuria.

Conclusions: DR affects one in five patients with DM in Brunei Darussalam, comparable to rates reported for other Asian populations. It is especially worrying that one in ten patients with DM had vision-threatening DR. DR was significantly associated with longer duration of DM, poor control and presence of microalbuminuria but reduced in older age groups. It is important to advocate good control right from the time of diagnosis of DM and institute timely and effective management of retinopathy. DR was significantly associated with longer duration of DM, poor control of diabetes, and presence of microalbuminuria but reduced in older age groups.

Key Words: Asians, Diabetic retinopathy, Prevalence, Risk factors, Type 2 diabetes mellitus

Received: March 19, 2021 Final revision: October 25, 2021

Accepted: October 28, 2021

Corresponding author: Sajid Adhi Raja, MS, FRCSEd. Department of Ophthalmology, Pengiran Muda Mahkota Pengiran Muda Haji Al-Muhtadee Billah Hospital, Tutong TA1341, Brunei Darussalam. Tel: 673-4260729, E-mail: sajid.adhiraja@moh.gov.bn
Diabetic retinopathy (DR) is a leading cause of blindness among adults worldwide [1]. It progresses in stages from mild to moderate, severe non-proliferative DR (NPDR) and proliferative DR (PDR) [2]. Vitreous hemorrhage in 
PDR is a cause of severe or sudden loss of vision. Macular oedema can occur in any stages of retinopathy and cause moderate loss of vision. Vision-threatening DR (VTDR) is defined as severe NPDR, PDR, or the presence of diabetic macular oedema [3].

With the increasing prevalence of non-communicable diseases, the number of people with diabetes mellitus (DM) and related complications is expected to increase. A decade ago, the overall global prevalence of DR was reported as $34.6 \%$ [4]. A more recent review of articles published between 2015 and 2018 reported a global prevalence of $27 \%$ and highlighted considerable heterogeneity in prevalence. The lowest prevalence was reported in Europe (20.6\%) and Southeast Asia (12.5\%) and highest in Africa (33.8\%), Middle East and North Africa (33.8\%), North America (34\%), and the Western Pacific region (36.2\%) [5]. This heterogeneity may be due to varying prevalence of DM and other non-communicable diseases such as obesity, as well as differences in awareness, level of knowledge and available medical facilities in different parts of the world.

The International Diabetic Federation estimates the worldwide prevalence of DM is $9.1 \%$ with type $2 \mathrm{DM}$ being the most common form [6]. Brunei Darussalam has an estimated prevalence of $13.3 \%$ [6]. The National Diabetic Management Programme of Brunei Darussalam mandates annual retinopathy screening for all patients with DM. To date, there have only been three studies published that had reported on prevalence of DR in Brunei Darussalam [7-9]. A study in 1998 at the Pengiran Muda Mahkota Pengiran Muda Haji Al-Muhtadee Billah Hospital, Tutong looked at DM control and reported a prevalence of DR of $18.0 \%$ (background DR, 17.1\%; vitreous hemorrhage, $0.6 \%$; and blindness, 0.3\%) [7]. Another study between 1997 and 2000 from the tertiary center in Brunei Darussalam reported prevalence of DR of 19\% (background DR, 15\%; DR with maculopathy, 4\%) [8]. The latest study done in 2014 to assess a new screening tool for DR in Brunei Darussalam reported a prevalence of DR at $18.5 \%$ including combined prevalence of severe DR and PDR of 3.7\% [9]. Since the purpose of that study was to assess a new screening tool, the results obtained may not be a true representation of the epidemiological situation. None of these three studies assessed associations with other factors. The purpose of our study is to assess the current prevalence of DR and associated factors in Brunei Darussalam and to compare with reported rates of other populations.

\section{Materials and Methods}

\section{Setting and study design}

Brunei Darussalam has a small population of 450,000 people living across its four districts and served by four government hospitals. There is also support from 15 health centers, ten clinics and 22 maternal and child healthcare facilities. All patients with DM are routinely screened for DR at government health facilities. There is currently no private ophthalmic facility that offers DR screening and follow-up. This study was conducted at one of the four district hospitals, serving a population of 52,700 (2019 estimation; Department of Economic Planning and Statistics, Brunei Darussalam). The demographic breakdown resembles the national breakdown [10]. It is the only facility in the district equipped with NW8F (Topcon Health Care, Tokyo, Japan) for fundus digital imaging and fluorescein angiography, and Nidek MC-500 Pascal laser (Nidek, Aichi, Japan) for retina screening and treatment. The Brunei Darussalam Health Information and Management System (Bru-HIMS) electronically links all government health facilities across the country. Patients requiring vitrectomy or intravitreal anti-vascular endothelial growth factor treatment are referred to a tertiary center where these treatments are available.

This is a non-interventional cross-sectional study which retrospectively looked at the prevalence of DR and factors associated with DR. The data was collected as a part of a planned improvement of ophthalmic services audit in the district. During this period, services were provided as per normal departmental protocol and there were no new treatments or interventions provided. Institutional review board approval was not required. Data collected were anonymized. A statistician unfamiliar with the study population then analyzed and curated the data. This study was conducted with adherence to the general principles of the Helsinki declaration 2008. All patients gave verbal consent for their data to be analyzed for the study.

\section{Patients and study participants}

Based on the population size and estimated prevalence of DM in Brunei Darussalam, the estimated burden of DM in the district is approximately 4,400 patients. All patients diagnosed with DM within the district are referred to the 
district hospital for assessment of visual acuity, non-contact intraocular pressure and retinal examination by non-mydriatic camera or by slit-lamp bio-microscopy if photographic images are not of gradable quality. Findings are recorded in the Bru-HIMS electronic database and further review appointments given based on the retinal findings; annually for patients with no retinopathy and 3 to 12 months for patients with DR, depending on severity. Fluorescein angiography is being done less frequently unless the patient has concurrent retinal vessel occlusions, suspected ischemic macular disease, or optic nerve head disease. Laser treatment for DR is offered in the eye clinic and patients requiring anti-vascular endothelial growth factor or vitrectomy are referred to the national referral center for treatment, whilst still maintaining an appointment for routine follow-up in the district hospital.

Sample size calculation was conducted prior to the study based on prevalence of DR in the Western Pacific region $(36.2 \%)$ and the estimated DM population $(4,400)$ in the district. A sample of 329 patients was required to provide a $95 \%$ confidence interval (CI) and 5\% margin of error. Patients with DM who attended the diabetic eye clinic for DR screening and monitoring over a 3-month period (October 7, 2019 to January 7, 2020) were recruited consecutively until sample size was achieved. In total, data of all patients ( $n=341)$ who attended screening or follow-up during the 3-month study was collected. The extracted data included age, sex, duration of DM, glycated hemoglobin (HbAlc) levels, presence of hypertension, hyperlipidemia, and microalbuminuria.

\section{DR screening outcome}

DR screening was done using the Topcon NW8F non-mydriatic camera. Retinal images for each patient are captured and stored in the patient database linked to the Bru-HIMS electronic record system. For patients with non-gradable quality digital images, a dilated fundus examination was conducted using a slit-lamp biomicroscope. DR was classified into five groups based on a simplified version of the Early Treatment of Diabetic Retinopathy Study classification. The five groups are no DR; mild NPDR, if there are only microaneurysms less than 20 in four quadrants; severe NPDR, if there are four quadrant intraretinal bleeding, venous beading in at least two quadrants, or intraretinal microvascular abnormality in at least one quadrant; moderate NPDR, which has features more than mild but less than severe NPDR; and lastly, presence of new vessels at the disc or elsewhere on the retina with or without vitreous hemorrhage classify as PDR.

\section{Statistics}

Descriptive and statistical analyses were carried out using IBM SPSS ver. 22.0 (IBM Corp., Armonk, NY, USA). Data of all patients $(n=341)$ were used for the descriptive analyses and data are presented as mean and standard deviations for continuous variables and absolute number and percentages for categorical variables including $95 \%$ CI. Ten patients with anemia were excluded as serum HbAlc is affected by this condition and hence may not reflect true DM control, leaving 331 patients for analyses. Age groups were categorized into under 40,41 to 50,51 to 60,61 to 70 , and over 70 years, with duration of DM in years into under 5,6 to 10,11 to 15,15 to 20, and over 20 years. DM control was categorized based on HbAlc level into good control $(\leq 7.5 \%)$, suboptimal ( $7.6 \%$ to $8.9 \%$ ) and poor control $(\geq 9.0 \%)$. Univariate analyses were conducted using the chisquare test and any variables found to be significant ( $p<$ 0.05 ) were entered into multivariate analysis.

\section{Results}

The largest age group was in the 51 to 60 years old range, with more females than males (58.9\%) and the population was predominantly Malay (consistent with the district and national population breakdown). Most patients had DM of less than 10 years (61.9\%), and the mean duration of DM was $9.4 \pm 7.4$ years. Thirty patients $(8.8 \%)$ had DM of less than one year. The mean serum HbAlc level was $8.5 \% \pm 1.9 \%$ with 150 patients $(44.0 \% ; 95 \% \mathrm{CI}$, $38.9 \%-49.4 \%$ ) categorized as having good control (HbAlc $\leq 7.5 \%)$. Hypertension (86.8\%) and dyslipidemia (84.5\%) were common and $22.6 \%$ had microalbuminuria or worst. Patients' demographics and profiles are shown in Table 1.

The prevalence of DR was $22.6 \%$ (95\% CI, 18.8-27.1). Among patients with DR, 14 patients (18.2\%; 95\% CI, 9.828.4) had PDR with overall prevalence of PDR being $4.1 \%$ (95\% CI, 2.1\%-6.5\%). Macular oedema occurred in 17 patients (5.0\%; 95\% CI, 2.9-7.7) and VTDR occurred in 9.7\% $(95 \%$ CI, $6.8 \%-13.3 \%)$ of all patients. The breakdown is 
Table 1. The patients' demographic and profiles $(n=341)$

\begin{tabular}{|c|c|c|}
\hline Variable & Value & $\begin{array}{c}95 \% \\
\text { confidence } \\
\text { interval }\end{array}$ \\
\hline Age (yr) & $55.3 \pm 11.9$ & $54.0-56.6$ \\
\hline$\leq 30$ & $12(3.5)$ & $1.8-5.5$ \\
\hline $31-40$ & $29(8.5)$ & $5.6-11.7$ \\
\hline $41-50$ & $65(19.1)$ & $15.1-23.5$ \\
\hline $51-60$ & $118(34.0)$ & $29.2-40.0$ \\
\hline $61-70$ & $92(27.10)$ & $22.1-31.5$ \\
\hline$>70$ & $25(7.43)$ & $4.7-10.4$ \\
\hline \multicolumn{3}{|l|}{ Sex } \\
\hline Male & $140(41.1)$ & $35.7-46.3$ \\
\hline Female & $201(58.9)$ & $53.7-64.3$ \\
\hline \multicolumn{3}{|l|}{ Ethnicity } \\
\hline Malays & $254(74.5)$ & $69.5-79.0$ \\
\hline Chinese & $21(6.1)$ & $3.9-9.3$ \\
\hline Indigenous & $66(19.4)$ & $15.3-24.0$ \\
\hline $\begin{array}{l}\text { Duration of diabetes } \\
\text { mellitus (yr) }\end{array}$ & $9.4 \pm 7.4$ & $8.6-10.2$ \\
\hline$\leq 5$ & $136(39.9)$ & $34.7-45.1$ \\
\hline $6-10$ & $75(22.0)$ & $17.5-26.6$ \\
\hline $11-15$ & $63(18.5)$ & $14.1-22.6$ \\
\hline $16-20$ & $33(9.7)$ & $6.7-12.9$ \\
\hline$>20$ & $34(10.0)$ & $7.0-13.2$ \\
\hline Glycated hemoglobin (\%) & $8.4 \pm 1.9$ & $8.2-8.6$ \\
\hline$\leq 6.9$ & $82(24.0)$ & $19.5-28.4$ \\
\hline $7.0-7.9$ & $84(24.6)$ & $20.1-29.4$ \\
\hline $8.0-8.9$ & $58(17.0)$ & $13.3-21.3$ \\
\hline $9.0-9.9$ & $53(15.5)$ & $11.7-19.5$ \\
\hline $10.0-10.9$ & $21(6.2)$ & $3.8-8.8$ \\
\hline $11.0-11.9$ & $24(7.0)$ & $4.7-9.9$ \\
\hline$\geq 12.0$ & $19(5.6)$ & $3.2-8.2$ \\
\hline \multicolumn{3}{|l|}{ Comorbidities } \\
\hline Hypertension (yes) & $296(86.8)$ & $82.9-90.1$ \\
\hline Dyslipidemia (yes) & $288(84.5)$ & $80.5-88.3$ \\
\hline Albuminuria (yes) & $77(22.6)$ & $18.8-27.1$ \\
\hline
\end{tabular}

Values are presented as mean \pm standard deviation or number (\%).

shown in Table 2.

In univariate analysis, age groups ( $p=0.030$ for trend), longer duration of $\mathrm{DM}$ ( $p=0.002$ for trend), DM control ( $p$ $<0.0001$ for trend) and presence of any microalbuminuria
Table 2. The breakdown of retina screening outcome $(\mathrm{n}=$ 341)

\begin{tabular}{lcc}
\hline Type of retinopathy & Value & $\begin{array}{c}95 \% \text { confidence } \\
\text { interval }\end{array}$ \\
\hline No DR & $263(77.1)$ & $72.7-81.9$ \\
Any DR & $78(22.9)$ & $18.8-27.1$ \\
$\quad$ Mild NPDR & $24(7.0)$ & $4.4-9.9$ \\
Moderate NPDR & $34(10.0)$ & $7.0-13.2$ \\
Severe NPDR & $6(1.8)$ & $0.6-3.2$ \\
PDR & $14(4.1)$ & $2.1-6.5$ \\
\hline
\end{tabular}

Values are presented as number (\%).

$\mathrm{DR}=$ diabetic retinopathy $\mathrm{NPDR}=$ non-proliferative DR; PDR $=$ proliferative $\mathrm{DR}$.

$(p<0.05)$ were significantly associated with DR (Table 3$)$. Sex, hypertension, and dyslipidemia did not show significant associations (all $p>0.05$ ). When these variables were entered into multivariate analysis, duration of DM, poor control of DM, and presence of microalbuminuria remained significantly associated with presence of any DR (Table 4). Duration of DM for 11 years or more, having poor DM control, and microalbuminuria were associated with any DR. Compared to the youngest age group $(<40$ years), the 61 to 70 and over 70 age groups had $80 \%$ to $90 \%$ lower risk for DR $(p=0.006)$.

\section{Discussion}

Our study showed that DR is common among patients with type $2 \mathrm{DM}$ in Brunei Darussalam with one in five affected and that certain variables were associated with the presence of DR. The prevalence of DR at $22.6 \%$ in our study is within the range reported across continents in the literature as shown in Table 5 [7-9,11-25]. Globally, reported rates range from those in Spain (12.3\%) [15], India (15.4\%) [18], and Korea (15.8\%) [24] to high rates reported in Indonesia (43.1\%) [23], Malaysia (39.3\%) [20], and Saudi Arabia (36.4\%) [17]. Studies from China, UK, and US all reported rates of above $30 \%$ [11,14,19]. Overall, there seems to be no definite correlations with the relative development of the countries. These variations can be accounted by several factors especially the patient population studied in these publications. Generally, studies tend to be done in 
Table 3. Univariate analyses between variables and presence or absence of any DR $(n=331)$

\begin{tabular}{|c|c|c|c|}
\hline Variable & Any DR & No DR & $p$-value \\
\hline \multicolumn{4}{|l|}{ Age group (yr) } \\
\hline$\leq 40$ & $11(27.5)$ & $29(76.7)$ & 0.030 for trend \\
\hline $41-50$ & $15(24.2)$ & $47(75.8)$ & \\
\hline $51-60$ & $36(30.8)$ & $81(69.2)$ & \\
\hline $61-70$ & $13(14.8)$ & $75(85.2)$ & \\
\hline$>70$ & $2(8.3)$ & $22(91.7)$ & \\
\hline \multicolumn{4}{|l|}{ Sex } \\
\hline Male & $31(23.0)$ & $104(77.0)$ & 0.915 \\
\hline Female & $46(23.5)$ & $150(76.5)$ & \\
\hline \multicolumn{4}{|c|}{ Duration of diabetes mellitus (yr) } \\
\hline$\leq 5$ & $19(14.7)$ & $110(85.3)$ & 0.002 for trend \\
\hline $6-10$ & $13(17.6)$ & $61(82.4)$ & \\
\hline $11-15$ & $22(36.1)$ & $39(63.9)$ & \\
\hline $16-20$ & $11(33.3)$ & $22(66.7)$ & \\
\hline$>20$ & $12(35.3)$ & $22(64.7)$ & \\
\hline \multicolumn{4}{|l|}{ Diabetes mellitus control } \\
\hline Good control & $20(14.3)$ & $120(85.7)$ & $<0.0001$ for trend \\
\hline Suboptimal & $15(20.3)$ & $59(79.7)$ & \\
\hline Poor & $42(35.9)$ & $75(64.1)$ & \\
\hline Hypertension (yes) & $67(87.0)$ & $222(87.4)$ & 0.928 \\
\hline Dyslipidemia (yes) & $68(88.3)$ & $215(84.6)$ & 0.424 \\
\hline Microalbuminuria (yes) & $48(62.3)$ & $106(41.7)$ & 0.001 \\
\hline
\end{tabular}

Values are presented as number (\%).

$\mathrm{DR}=$ diabetic retinopathy.

academic or large centers and this can result in over representations of more severe cases. Milder or controlled cases are usually followed up in peripheral clinics and such patients may not be included in the population samples. Availability of healthcare and its' infrastructure are also important. With greater accessibility, the proportion of patients diagnosed with DM early is higher and the proportion of undiagnosed cases is expected to be lower. On the other hand, in less developed countries where prevalence of obesity is low or just beginning to increase, the prevalence of DM may similarly be low or just increasing. Therefore, the true prevalence of complications related to diabetes such as DR is likely to be lower in less developed countries when compared to developed countries. Despite this, reported figures represent major universal public health concern with one in eight to almost one in two having DR [11-26]. This is particularly important given that
DR is the main cause of visual impairment and blindness in adults and that the prevalence of DM is increasing [1].

Similarly, there is also heterogeneity in the reported prevalence of VTDR globally (Table 5) [7-9,11-25]. Interestingly, the prevalence of VTDR is generally low in developed nations and high in developing countries. This may be related to better control of DM and timely treatment of DR in the more developed world. On the other hand, changing food habits, increasing prevalence of non-communicable diseases such as obesity, hypertension, and dyslipidemia, and non-availability of timely and appropriate treatment of retinopathy may be reasons for increased rates of VTDR in developing countries. Further research is required to ascertain the reasons.

The current rate of $22.9 \%$ for any DR in Brunei Darussalam is slightly higher than the rates $(18.0 \%-19.0 \%)$ reported in the previous local studies from nearly two de- 
Table 4. Multivariate analyses of association with any diabetic retinopathy $(\mathrm{n}=331)$

\begin{tabular}{|c|c|c|c|c|}
\hline Variable & Number & Odds ratio & $p$-value & $95 \%$ confidence interval \\
\hline \multicolumn{5}{|l|}{ Age group (yr) } \\
\hline$\leq 40$ & 40 & Reference & & \\
\hline $41-50$ & 62 & 0.63 & 0.348 & $0.230-1.67$ \\
\hline $51-60$ & 117 & 0.76 & 0.545 & $0.320-1.84$ \\
\hline $61-70$ & 88 & 0.22 & 0.006 & $0.076-0.65$ \\
\hline$\geq 71$ & 24 & 0.09 & 0.007 & $0.020-0.52$ \\
\hline \multicolumn{5}{|c|}{ Duration of diabetes mellitus (yr) } \\
\hline$\leq 5$ & 129 & Reference & & \\
\hline $6-10$ & 74 & 1.53 & 0.301 & $0.68-3.47$ \\
\hline $11-15$ & 61 & 3.88 & 0.001 & $1.78-8.46$ \\
\hline $16-20$ & 33 & 3.39 & 0.014 & $1.23-8.98$ \\
\hline$>20$ & 34 & 5.43 & 0.002 & $1.89-15.59$ \\
\hline \multicolumn{5}{|c|}{ Diabetes mellitus control } \\
\hline Good & 140 & Reference & & \\
\hline Suboptimal & 74 & 1.14 & 0.753 & $0.51-2.51$ \\
\hline Poor & 117 & 2.01 & 0.039 & $1.03-3.90$ \\
\hline \multicolumn{5}{|c|}{ Microalbuminuria } \\
\hline No & 177 & Reference & & \\
\hline Yes & 154 & 2.00 & 0.019 & $1.12-3.59$ \\
\hline
\end{tabular}

cades ago [7-9]. The combined rate of severe DR and PDR $(6.3 \%)$ in the current study is also higher than the last study of $3.7 \%$ carried out more than 5 years ago [9]. The study by Da and Nyunt [7] which was conducted in the same institution as the current study provided a unique opportunity for comparison. The proportion of patients with good control of DM (HbA1c $<8.0 \%$ ) has declined from $48.6 \%$ in the year 2003 to $60.1 \%$ at present. In contrast, the proportion of patients with hypertension has increased from $61.7 \%$ to $86.8 \%$ and likewise dyslipidemia has increased, reflecting changes to the patient demographic over time [7]. Retinopathy has increased from $18.0 \%$ to $22.9 \%$. These differences may be a true reflection of increasing prevalence of DR in Brunei Darussalam. Perhaps the prevalence of non-communicable diseases including DM has been increasing with greater awareness and understanding, contributing to the increasing number of patients being diagnosed with DM.

Our study showed several factors to be significantly associated with DR. Among the known risk factors for development of DR, duration of DM is the most important non-modifiable factor. According to large epidemiological studies, the risk of developing DR increased several folds after a latent period of 8 to 10 years' duration of DM $[4,25]$. We showed that patients who had DM of more than 10 years were significantly associated with DR: 11 to 15 years (odds ratio [OR], 3.88), 16 to 20 years (OR, 3.39) and over 20 years (OR, 5.43). Among the patients in this study, $61.9 \%$ have DM of less than 10 years duration. This indicates a likely increase in the prevalence of DR in the population in the coming years.

In the management of DM, good control of diabetes is very important to reduce the risk of future complications. However, even with good control, complications do occur due to the chronic nature of the disease. Our study showed a linear correlation between DR and levels of control: good control (14.3\%), suboptimal control $(20.3 \%)$ and poor control (35.9\%). Poor control was significantly associated with development of DR (OR, 2.01). The American Diabetes Association recommends $7 \%$ as target of HbAlc level for good control [26]. The Diabetes Control and Complications Trial and the United Kingdom Prospective Diabetes Study showed that increasing levels of HbAlc correlated well with occurrence and progression of DR [27,28]. However, 
Table 5. Comparison of prevalence of diabetic retinopathy among diabetes patients in Brunei Darussalam with some recently published data from countries around the world

\begin{tabular}{|c|c|c|c|c|c|c|c|}
\hline \multirow{2}{*}{ Study } & \multirow{2}{*}{ Country } & \multirow{2}{*}{ Sample size } & \multirow{2}{*}{$\begin{array}{l}\text { Mean age } \\
(\mathrm{yr})\end{array}$} & \multirow{2}{*}{ DR $(\%)$} & \multicolumn{3}{|c|}{ Vision-threatening DR (\%) } \\
\hline & & & & & Severe NPDR & PDR & DME \\
\hline Naik et al. (2018) [11] & USA & 5,242 & NR & 34.0 & 1.6 & 1.2 & 4.3 \\
\hline Kanjee et al. (2016) [12] & Canada & 4,338 & 53.0 & 25.1 & NR & 6.5 & 0.2 \\
\hline Vujosevic et al. (2017) [13] & Italy & 8,596 & NR & 27.5 & 2.9 & 0.9 & 5.7 \\
\hline Thomas et al. (2015) [14] & UK & 8,6390 & 65.3 & 30.3 & NR & 0.31 & NR \\
\hline $\begin{array}{l}\text { Rodriguez-Poncelas et al. } \\
\text { (2015) [15] }\end{array}$ & Spain & 10,8723 & 66.9 & 12.3 & 0.86 & 0.36 & 0.18 \\
\hline Dehghan et al. (2015) [16] & Iran & 529 & 44.0 & 29.6 & 3.9 & 3.9 & 4.9 \\
\hline Ahmed et al. (2016) [17] & Saudi Arabia & 401 & 54.5 & 36.4 & 11.0 & 11.6 & 7.2 \\
\hline Sunita et al. (2018) [18] & India & 592 & 51.0 & 15.4 & NR & 6.7 & $6.6^{*}$ \\
\hline Liu et al. (2017) [19] & China & 13,473 & 62.5 & 34.1 & 0.89 & 11.5 & NR \\
\hline $\begin{array}{l}\text { Abougalambou SS and } \\
\text { Abougalambou AS (2014) [20] }\end{array}$ & Malaysia & 1,077 & NR & 39.3 & NR & NR & NR \\
\hline Tan et al. (2018) [21] & Singapore & 2,862 & 61.6 & 33.9 & 0.71 & 3.75 & 7.6 \\
\hline Jongsareejit et al. (2013) [22] & Thailand & 1,120 & NR & 24.0 & NR & 2.8 & NR \\
\hline Sasongko et al. (2017) [23] & Indonesia & 1,138 & 59.0 & 43.1 & 11.1 & 12.1 & 17.1 \\
\hline Jee et al. (2013) [24] & Korea & 1,678 & 63.0 & 15.8 & 0.8 & 2.8 & 4.5 \\
\hline Current study (2020) & Brunei Darussalam & 341 & 55.3 & 22.9 & 1.5 & 4.7 & 5.0 \\
\hline Da and Nyunt (2003) [7] & Brunei Darussalam & 358 & NR & 18.0 & NR & NR & NR \\
\hline Joshi et al. (2003) [8] & Brunei Darussalam & 5,365 & NR & 19.0 & NR & NR & 4.0 \\
\hline Ali et al. (2015) [9] & Brunei Darussalam & 1,184 & NR & 18.5 & $3.7^{\dagger}$ & NR & 6.4 \\
\hline
\end{tabular}

Values are presented as number.

$\mathrm{DR}=$ diabetic retinopathy; NPDR $=$ non-proliferative $\mathrm{DR} ; \mathrm{PDR}=$ proliferative $\mathrm{DR} ; \mathrm{DME}=$ diabetic macular oedema; $\mathrm{NR}=$ not recorded.

${ }^{*}$ Clinically significant DME. ${ }^{\dagger}$ Includes PDR.

later studies demonstrated that strict control of DM does not give clear benefits in controlling macro-vascular diseases, unlike in microvascular diseases [29-31]. The Action to Control Cardiovascular Risk in Diabetes study showed that intensive control of DM increased the risk of mortality especially in type 2 DM [32]. In our setting, we use HbAlc $\leq 7.5 \%$ as a target value for good control. Later analysis of the above mentioned and similar studies suggest that HbAlc targets should be individualized based on the duration of the DM and existing comorbidities [33]. Generally, younger individuals with DM who are otherwise healthy should aim for a lower target value whereas older individuals with cardiovascular comorbidities should have a higher target level of HbAlc.

Albuminuria in DM is a wide spectrum ranging from micro to macroalbuminuria, which usually correlates with duration and severity of the disease. It is an important predictor of DM nephropathy. Not unexpectedly, our study showed a positive association between microalbuminuria and DR (OR, 2.00). Therefore, it is important that interval screening for microalbuminuria be carried out.

Interestingly, we found that among the age groups, the older patients had a lower risk for DR, (61-70 years group with OR $0.22, p=0.006$ and $>70$ years group with OR 0.09 , $p=0.007$ ) when compared to the over 40 years group. This probably indicates for uncertain reason that the younger age groups were at higher risk for DR. At first sight, this may seem contradictory to our finding that a longer duration of DM was significantly associated with DR. We found that the older groups (61-70 and $>70)$, despite having a longer duration of DM, had better mean HbAlc levels and good DM control when compared to the younger age 
groups. Furthermore, factors that were not considered in our study may contribute, such as genetic predisposition and lifestyle. The phenomenon of young DM patients having more complications is probably a reflection of the changing demographic in DM, with more young people developing DM, corresponding with the increasing prevalence of obesity and metabolic syndromes.

Among the factors assessed, hypertension and hyperlipidemia were not associated with DR. Hypertension can cause retinopathy and nephropathy and is often correlated with severe and chronic hypertension. In DM, the management of hypertension is more aggressive with lower target levels making contribution of hypertension less important than DM itself $[34,35]$. There have been several studies examining the effect of hypertension and hyperlipidemia in relation to diabetic complications. A meta-analytic review published in Cochrane patients concluded that there is only weak evidence for beneficial effects of reduction in blood pressure with respect to preventing DR [36]. The Action to Control Cardiovascular Risk in Diabetes Eye Study showed that intensive glycemic control together with control of dyslipidemia using fenofibrate and simvastatin reduced the proportion of eyes that had progression of DR by one-third [37]. However, the beneficial effect may be due to the intensive management of DM rather than lipid control. Similar to the management of hypertension, the recommendation for initiating treatment for dyslipidemia in patients with DM is also more aggressive. All our patients with diagnosis of hypertension and hyperlipidemia were receiving treatment.

There are several limitations to our study that need to be considered when interpreting the study findings. First, our study was a retrospective study and there are inherent limitations with retrospective studies such as incomplete data and selection bias. Our study looked into already known risk factors for DR in the context of Brunei Darussalam. Second, this study was conducted in a single hospital and might not accurately represent the whole country. However, the patient population breakdown in our study is consistent with that of the national breakdown and previous studies from different districts have reported comparable results $[7,8]$. Third, we only included patients over a 3-month period, and it is possible that more patients with significant DR would be included, given their shorter follow-up intervals. This may inflate the prevalence of DR. However, the total number of patients with significant
PDR who will have shorter follow-up interval of 3 months is small. Fourth, our study population excluded patients with complicated diseases such as diabetic foot disease, cardiac, or renal disease, as they typically received treatment and are followed up in the tertiary hospital. Such patients are likely to have DR and not captured in our study. Hence, the prevalence of DR could be higher than our estimate. However, the proportion of such patients is estimated to be small.

We showed that DR in Brunei Darussalam is common with a rate of $22.6 \%$, which is comparable to what has been reported in the literature. DR affects one in five patients with type $2 \mathrm{DM}$ in Brunei Darussalam, comparable to rates reported for other Asian populations. A third of those with DR had either PDR or VTDR. Longer duration of DM, poor control of diabetes, and presence of microalbuminuria are significantly associated with DR. Interestingly, age groups were not linearly associated with DR, and this requires further study. Since a large percentage of DR occurs in young and middle-aged patients, it is worthwhile to advocate strict control of DM from the outset. Public and targeted awareness programs are needed, especially in countries with young and growing population, like Brunei Darussalam.

\section{Conflicts of Interest}

No potential conflict of interest relevant to this article was reported.

\section{Acknowledgements}

None.

\section{Funding}

The authors received no financial support for this article.

\section{References}

1. GBD 2019 Blindness and Vision Impairment Collaborators; Vision Loss Expert Group of the Global Burden of Disease Study. Trends in prevalence of blindness and distance and 
near vision impairment over 30 years: an analysis for the Global Burden of Disease Study. Lancet Glob Health 2021;9:e130-43.

2. Fong DS, Aiello L, Gardner TW, et al. Retinopathy in diabetes. Diabetes Care 2004;27 Suppl 1:S84-7.

3. Lee R, Wong TY, Sabanayagam C. Epidemiology of diabetic retinopathy, diabetic macular edema and related vision loss. Eye Vis (Lond) 2015;2:17.

4. Yau JW, Rogers SL, Kawasaki R, et al. Global prevalence and major risk factors of diabetic retinopathy. Diabetes Care 2012;35:556-64.

5. Thomas RL, Halim S, Gurudas S, et al. IDF Diabetes Atlas: a review of studies utilising retinal photography on the global prevalence of diabetes related retinopathy between 2015 and 2018. Diabetes Res Clin Pract 2019;157:107840.

6. International Diabetes Federation. IDF DM Atlas 2019 [Internet]. Brussels: International Diabetes Federation; 2019 [cited 2021 Jan 17]. Available from: https://diabetesatlas. org/resources/.

7. Da PT, Nyunt K. Study of diabetic management, control and complications among the patients attending diabetic clinic at PMMPMHAMB Hospital, Tutong district, Brunei Darussalam. Brunei Int Med J 2003;3:33-8.

8. Joshi N, Mathew R, Abdul Momin N. Incidence of diabetic retinopathy in Brunei Darussalam. Brunei Int Med J 2003;3:3941.

9. Ali NA, George J, Joshi N, Lim MS, Siraj AA. Findings of diabetes eye screening in Brunei Darussalam: REPAS diabetic retinopathy grading scale. Brunei Int Med J 2015;11:182-90.

10. Department of Economic Planning and Statistics. Brunei population snapshot mid-year population estimates 2019 [Internet]. Bandar Seri Begawan: Department of Economic Planning and Statistics, Ministry of Finance and Economy; 2019 [cited 2021 Jan 17]. Available from: http://www.deps. gov.bn/.

11. Naik S, Wykoff CC, Ou WC, et al. Identification of factors to increase efficacy of telemedicine screening for diabetic retinopathy in endocrinology practices using the Intelligent Retinal Imaging System (IRIS) platform. Diabetes Res Clin Pract 2018;140:265-70.

12. Kanjee R, Dookeran RI, Mathen MK, et al. Six-year prevalence and incidence of diabetic retinopathy and cost-effectiveness of tele-ophthalmology in Manitoba. Can J Ophthalmol 2016;51:467-70.

13. Vujosevic S, Pucci P, Casciano M, et al. A decade-long telemedicine screening program for diabetic retinopathy in the north-east of Italy. J Diabetes Complications 2017;31:134853.

14. Thomas RL, Dunstan FD, Luzio SD, et al. Prevalence of diabetic retinopathy within a national diabetic retinopathy screening service. Br J Ophthalmol 2015;99:64-8.

15. Rodriguez-Poncelas A, Miravet-Jimenez S, Casellas A, et al. Prevalence of diabetic retinopathy in individuals with type 2 diabetes who had recorded diabetic retinopathy from retinal photographs in Catalonia (Spain). Br J Ophthalmol 2015;99: 1628-33.

16. Dehghan $\mathrm{MH}$, Katibeh M, Ahmadieh H, et al. Prevalence and risk factors for diabetic retinopathy in the 40 to 80 year-old population in Yazd, Iran: the Yazd Eye Study. $J$ Diabetes 2015;7:139-41.

17. Ahmed RA, Khalil SN, Al-Qahtani MA. Diabetic retinopathy and the associated risk factors in diabetes type 2 patients in Abha, Saudi Arabia. J Family Community Med 2016;23:1824.

18. Sunita M, Singh AK, Rogye A, et al. Prevalence of diabetic retinopathy in urban slums: the Aditya Jyot diabetic retinopathy in urban Mumbai slums study-report 2. Ophthalmic Epidemiol 2017;24:303-10.

19. Liu Y, Song Y, Tao L, et al. Prevalence of diabetic retinopathy among 13473 patients with diabetes mellitus in China: a cross-sectional epidemiological survey in six provinces. BMJ Open 2017;7:e013199.

20. Abougalambou SS, Abougalambou AS. Risk factors associated with diabetic retinopathy among type 2 diabetes patients at teaching hospital in Malaysia. Diabetes Metab Syndr 2015;9:98-103.

21. Tan GS, Gan A, Sabanayagam C, et al. Ethnic differences in the prevalence and risk factors of diabetic retinopathy: the Singapore Epidemiology of Eye Diseases Study. Ophthalmology 2018;125:529-36.

22. Jongsareejit A, Potisat S, Krairittichai U, et al. The Thai DMS Diabetes Complications (DD.Comp.) project: prevalence and risk factors of diabetic retinopathy in Thai patients with type 2 diabetes mellitus. J Med Assoc Thai 2013;96:1476-82.

23. Sasongko MB, Widyaputri F, Agni AN, et al. Prevalence of diabetic retinopathy and blindness in Indonesian adults with type 2 diabetes. Am J Ophthalmol 2017;181:79-87.

24. Jee D, Lee WK, Kang S. Prevalence and risk factors for diabetic retinopathy: the Korea National Health and Nutrition Examination Survey 2008-2011. Invest Ophthalmol Vis Sci 2013;54:6827-33. 
25. Huang OS, Tay WT, Ong PG, et al. Prevalence and determinants of undiagnosed diabetic retinopathy and vision-threatening retinopathy in a multiethnic Asian cohort: the Singapore Epidemiology of Eye Diseases (SEED) study. Br J Ophthalmol 2015;99:1614-21.

26. American Diabetes Association. Standards of medical care in diabetes: 2013. Diabetes Care 2013;36 Suppl 1(Suppl 1):S11-66.

27. Progression of retinopathy with intensive versus conventional treatment in the Diabetes Control and Complications Trial. Diabetes Control and Complications Trial Research Group. Ophthalmology 1995;102:647-61.

28. Stratton IM, Adler AI, Neil HA, et al. Association of glycaemia with macrovascular and microvascular complications of type 2 diabetes (UKPDS 35): prospective observational study. BMJ 2000;321:405-12.

29. Albers JW, Herman WH, Pop-Busui R, et al. Effect of prior intensive insulin treatment during the Diabetes Control and Complications Trial (DCCT) on peripheral neuropathy in type 1 diabetes during the Epidemiology of Diabetes Interventions and Complications (EDIC) Study. Diabetes Care 2010;33:1090-6.

30. Kirkman MS, McCarren M, Shah J, et al. The association between metabolic control and prevalent macrovascular disease in Type 2 diabetes: the VA Cooperative Study in diabetes. J Diabetes Complications 2006;20:75-80.
31. ADVANCE Collaborative Group, Patel A, MacMahon S, et al. Intensive blood glucose control and vascular outcomes in patients with type 2 diabetes. $N$ Engl J Med 2008;358:256072.

32. Action to Control Cardiovascular Risk in Diabetes Study Group, Gerstein HC, Miller ME, et al. Effects of intensive glucose lowering in type 2 diabetes. $N$ Engl J Med 2008;358:254559.

33. Terry T, Raravikar K, Chokrungvaranon N, Reaven PD. Does aggressive glycemic control benefit macrovascular and microvascular disease in type 2 diabetes?: insights from ACCORD, ADVANCE, and VADT. Curr Cardiol Rep 2012;14:79-88.

34. Chang $\mathrm{CH}$, Chuang LM. Microalbuminuria and diabetic retinopathy in type 2 diabetic patients: from risk association to risk prediction. J Diabetes Investig 2013;4:42-4.

35. Rajalakshmi R, Shanthi Rani CS, Venkatesan U, et al. Correlation between markers of renal function and sight-threatening diabetic retinopathy in type 2 diabetes: a longitudinal study in an Indian clinic population. BMJ Open Diabetes Res Care 2020;8:e001325.

36. Do DV, Wang X, Vedula SS, et al. Blood pressure control for diabetic retinopathy. Cochrane Database Syst Rev 2015;1:CD006127.

37. Frank RN. Systemic therapies for diabetic retinopathy: the accord eye study. Ophthalmology 2014;121:2295-6. 